\title{
Combined Multi-Encryption Techniques for Text Securing Using Block Cipher and Stream Cipher Crypto-Systems
}

\author{
http://dx.doi.org/10.3991/ijes.v1i1.2901 \\ Hassene seddik \\ University of Tunis, Tunis, Tunisia
}

\begin{abstract}
Due to the growth of electronic intrusion techniques and personal data control, it is necessary for private or public organization to secures their data and prove their owner rights. Encryption techniques provide a useful mean to secure internet files exchange or numeric storage data. Old techniques present some disadvantages decreasing their robustness face to attacks. In this paper a new approach presenting a multi-encryption system for text and file text is presented. Dividing the file in different parts, each part is encrypted by a different algorithm to generate a polyencrypted file.
\end{abstract}

Index Terms-Block encryption, stream cipher encryption, poly-encryption, combined encryption, text and file encryption.

\section{INTRODUCTION}

Many encryption software and applications are freely downloadable on the internet for text and file text securing. For example the software named "cryptage-text" available at this link: http://www.softonic.fr/s/cryptage-texte allows these tasks with the most absolute ease [1]. However, all these programs are based on algorithms whose attacks strategies are previously known [2]. It is in this context that we decided to develop an approach inspired on existing algorithms but by exploiting their advantages and improving their unseemly to increase their strength against different possible attacks. In this paper two encryption techniques are developed. They are combined alternately to encrypt pseudo-randomly a file. This file will be decomposed into sub-spaces based on the number of words that it contains, and each subset is assigned to a chosen encryption technique. Once all the text is encrypted it will be grouped again to present a secured file.

\section{PROPOSED ENCRYPTION TECHNIQUES}

In this paper two encryption techniques are proposed. Based on two families of symmetric cryptoSystems: combined block cipher and stream cipher. The development of these algorithms is inspired from in use techniques exploiting their improper to make them more robust and more resistant to preconceived attacks. The first technique is called Logic Stream Cipher Encryption "LSCE" which is based on the logic function "xor", and the second is called "DESM" which is a modified version of the famous algorithm "DES" [3].

\section{A. Presentation of the LSCE Encryption technique}

The first proposed technique is an algorithm that belongs to the family of encryption stream or "stream cipher" symmetric key based on XOR logical operator that has a mathematical characteristic [4], the plaintext can be deduced from the encrypted and vice versa through the flow key by logical sum as shown in the following equations:

$$
x \oplus k=y
$$

$y$ and $x$ are respectively the cipher text and the plaintext, the operator $\oplus$ is an exclusive OR (XOR), $k$ the, key stream. Decryption is defined as follows:

$y \oplus k=(x \oplus k) \oplus k=x \oplus(k \oplus k)=x$

- The proposed approach differs from other approaches of the same types with the following advantages:

The generation of the encryption key as a random alphanumeric string drawn randomly with the same length as the original message. This key is generated from a short key belonging to the operator. The use of a short key is very important because it hides information about the actual size of the clear message.

- Increase the size of the dictionary based messages to 144 characters in extended ASCII noted $\mathfrak{\Im}$. Therefore extending the workspace is defined by the following equation: $\forall x \in \mathfrak{S}, e_{z}(x)=y / y \in \mathfrak{S}$

Stages of the creation of the key stream are achieved by the following method through a random short key input by the authorized user note $K_{o p}$ with length m:

$$
K_{o p}=\left\{k_{1} \ldots . k_{m}\right\}
$$

$K_{o p}$ is repeated a-periodically by reversing the order of its bits at each step P up to the end of the clear message. The step $\mathrm{P}$ is determined by the order of the letter of lowest weight 'LSB' of the $\mathrm{m}$ key elements introduced to generate the key space $\beta_{i}$. 
$\beta_{i}=\left\{\begin{array}{l}{\left[k_{1} \ldots k_{m}\right]_{1}, \ldots,\left[k_{1} \ldots . k_{m}\right]_{p},\left[k_{m} \ldots . k_{1}\right]_{1},\left[k_{m} \ldots . k_{1}\right]_{2}, \ldots} \\ ,\left[k_{m} \ldots . k_{1}\right]_{2 p}, \ldots . .,\left[k_{1} \ldots . k_{m}\right]_{i p}\end{array}\right\}$

Such that the value of the permutation $\mathrm{P}$ is not described as follows:

$$
\begin{aligned}
p= & L S B\left(k_{1} \ldots k_{m}\right) \\
& \beta_{i}=\left\{K_{1 o p}, K_{2 o p}, \ldots, k_{i o p}\right\}
\end{aligned}
$$

A function noted $\mathrm{C}$ is used to order the elements and truncate the flow order to $\mathrm{L}$ elements respectively to the size of the clear message.

$$
\begin{gathered}
\beta_{i+1}=C\left(\beta_{i}\right) \\
\beta_{L}=\left\{k_{1} \ldots k_{m}, k_{1} \ldots k_{m}, k_{1} \ldots k_{m}, \ldots, k_{1} \ldots k_{L}\right\}
\end{gathered}
$$

A permutation function $\mathrm{S}$ allows a redistribution of bits of the key of length $L$ by changing the position of its components depending on the position of the Kop vector elements beginning from the middle:

$$
Z_{i}=S_{i}\left(\beta_{L}, K_{i o p}\right)
$$

The randomness is introduced to break the correlated model of the function that models the periodicity of the key chain using the following equation

$$
\forall k \in K_{o p} \text {, and } \mathrm{Z}_{i}=S_{i}\left(\beta_{L}\right) \nexists f \text { such as } \mathrm{Z}_{i}=f\left(K_{i_{o p}}\right)
$$

Finally we recover a stream of random keys $\mathrm{Zi}$, which used to encrypt the original text.

$$
Z_{i}=\left\{k_{1}, k_{2}, k_{9}, k_{6}, k_{L-2} \ldots . . k_{L}\right\}(11)
$$

Our technique belongs to the families called onetime pad "OTP", since the key-stream $\mathrm{z}$ is generated randomly and independently of the text and the same length as the text to be encrypted. The encryption function $e_{k}(x)$ defines the cipher text by the following equation.

$$
e_{k}(x)=y_{i}=x_{i} \oplus z_{i}
$$

As $\quad Z_{i} \in \mathfrak{s}, \oplus$ is the logic operator (XOR), $y_{i}$ the encrypted text with length i, $x_{i}$ the plain text, $Z_{i}$ the key stream. Since the encryption technique is reversible and lossless then:

$\forall x \in \mathfrak{S}, \exists e_{z}(x) / h(z, x)=e_{z}(x)=y$ and $d_{z}(y)=h^{-1}(z, y)=x$ Then the steps of the decryption techniques are similar to those of the encryption ones, by replacing $y_{i}=h\left(z_{i}, x_{i}\right)$ par $x_{i}=h^{-1}\left(z_{i}, y_{i}\right)$ as shown by the following equation

$$
d_{k}(x)=x_{i}=y_{i} \oplus z_{i}
$$

The key-stream is independent from the plaintext and expanded space from 26 letters to the 144 ASCII characters. Since there is an independence from the keys flow and the plaintexts and encrypted one, the proposed technique is considered synchronous flow, described by the triplet following equation:

$$
\beta_{i+1}=C\left(\beta_{i}, K_{o p}\right) \quad z_{i}=S\left(\beta_{i}, K_{\text {iop }}\right) \quad y_{i}=h\left(z_{i}, x_{i}\right)(14)
$$

\section{B. DESM-block cipher algorithm based on the technique modified DES}

The second proposed algorithm is presented in the føHowing section and participates in alternating the poly-encryption of a file is an evolution towards nonconventional approaches based on a synchronous block encryption. These algorithms derive their scrambling operation of data Feistels networks deemed by their permutations generating a statistical confusion processed text. The proposed algorithm is a Feistel network with 160 rounds, with key k 56-bit generating diversified 160 keys of 48-bit encoding blocks of 64 bits. It uses substitution boxes (S-box) fixed to introduce confusion. The proposed method is based on the structure of the DES algorithm modified to generate a new algorithm called DESM [5].

\section{1) Steps of the proposed algorithm}

The algorithm we propose, which is based on the structure of DES is a symmetric encryption algorithm block which can encrypt word of 64 bits from one key of 56 bits ( 56 bits used to encrypt, the +8 parity bits used to verify the integrity of the key). We keep the same structure but the permutation, transposition, the number of rounds and the number of generated random key will be changed [6].

\section{2) Dynamic Partitioning permutation}

We begin by splitting the message "plain text" which gives the partitioning into blocks of 64 bits followed by a dynamics transposition of each block unlike the DES algorithm whose implementation is static. A function of four successive rotations increment is applied to each block to change the position of its transposed elements. The first rotation is applied to the fourths central bits of the initial permutation table, the second rotation processes 16 bits excluding the central quad already permutated bits giving 12 bits processed. The third rotation rotates thirty six bits minus the central core 16 bits which gives 20 bits processed. While the fourth pivots all 64 bits of the initial permutation table excluding the table core of 36 bits which returns to pivot on the 28 bit located on the borders of the initial permutation table. The technique of permutation and quad successive rotations are shown by the following figures and equations. Let $T(x, y) / x \in[1: 8]$ and $y \in[1: 8]$ the initial permutation table and a function $\mathrm{f}$ composed of 
four sub-functions such as $f=\left\{f_{1}, \ldots, f_{i}\right\} / i \in[1: 4]$. We consider the origin of the permutation table as the origin of the axes on the table while the rotations of the sub-matrices of the table will be made in the anticlockwise as follows:

$$
\begin{aligned}
& T_{1}(x, y)=\sum_{x=4}^{5} \sum_{y=4}^{5} T(x, y) \\
& T_{2}(x, y)=\sum_{x=3}^{6} \sum_{y=3}^{6} T(x, y)-\sum_{x=4}^{5} \sum_{y=4}^{5} T(x, y) \\
& T_{3}(x, y)=\sum_{x=2}^{7} \sum_{y=2}^{7} T(x, y)-\sum_{x=3}^{6} \sum_{y=3}^{6} T(x, y) \\
& T_{4}(x, y)=\sum_{x=1}^{8} \sum_{y=1}^{8} T(x, y)-\sum_{x=2}^{7} \sum_{y=2}^{7} T(x, y)
\end{aligned}
$$

The sub-functions of rotation is made by a step $\mathrm{P}$. the following figures show a rotation with a step of $\mathrm{P}$ $=1$. The transformation equations are as follows:

$$
\begin{aligned}
& T_{1}^{\prime}(x, y)=f_{1}^{P 1}\left(T_{1}(x, y)\right) \\
& T^{\prime}{ }_{2}(x, y)=f_{2}^{P 2}\left(T_{2}(x, y)\right) \\
& T^{\prime}{ }_{3}(x, y)=f_{3}^{P 3}\left(T_{3}(x, y)\right) \\
& T^{\prime}{ }_{4}(x, y)=f_{4}^{P 4}\left(T_{4}(x, y)\right)
\end{aligned}
$$

For each block of 64 bits the dynamic permutation table changes. This change will continue until all the blocks composing the whole message are transposed as described by the following equations

$$
\begin{aligned}
& T_{\text {Blck }_{1}}^{\prime}(x, y)=f^{P 1}(T(x, y)) \\
& T_{\text {Blck }_{2}}^{\prime}(x, y)=f^{P 2}\left(T^{\prime}{ }_{\text {Blck }_{1}}(x, y)\right) \\
& T_{\text {Blck }_{n}}^{\prime}(x, y)=f^{P(n-4 \times K+1)}\left(T_{\text {Blck }_{n-1}}^{\prime}(x, y)\right)
\end{aligned}
$$

While $K \in \mathcal{N}$ is the last integer that divides $\mathrm{n}$.

\begin{tabular}{|l|l|l|l|l|l|l|l|}
\hline 58 & 50 & 42 & 34 & 26 & 18 & 10 & 2 \\
\hline 60 & 52 & 44 & 36 & 28 & 20 & 12 & 4 \\
\hline 62 & 54 & 46 & 38 & 30 & 22 & 14 & 6 \\
\hline 64 & 56 & 48 & 40 & 32 & 24 & 16 & 8 \\
\hline 57 & 49 & 41 & 33 & 25 & 17 & 9 & 1 \\
\hline 59 & 51 & 43 & 35 & 27 & 19 & 11 & 3 \\
\hline 61 & 53 & 45 & 37 & 29 & 21 & 13 & 5 \\
\hline 63 & 55 & 47 & 39 & 31 & 23 & 15 & 7 \\
\hline
\end{tabular}

Figure 1. Initial permutation Table and its four cores

- blocks partition

Blocks are then divided into two blocks of 32 bits and noted $G$ and $D$. We note by $G_{0}$ and $D_{0}$ the initial state of the two blocks.

\section{- Expansion Function}

The 32 bits of the $D_{0}$ block are extended to 48 bits with a table of expansion in which 32 bits are mixed together and 16 are duplicated. All bits are duplicated and dispersed to create a block of 48 bits that will be called $D_{0}$ :

$$
B_{i}=\left\{\begin{array}{l}
b_{1}, b_{4}, b_{5}, b_{8}, b_{9}, b_{12}, b_{13}, b 16, b_{17}, b_{21}, b_{22}, \\
b_{24}, b_{25}, b_{28}, b_{29} \text { and } b_{32}
\end{array}\right\}
$$

- Logical Sum with the encryption key 48 bit

This is followed by applying XOR between $D_{0}$ and the first key $K_{1}$ generated from the key $K$ (that must be shared transmitter and receiver) by the timing algorithm of the key which will be described below. We call $D_{0}$ the result of this operation.

- substitution box

\begin{tabular}{|c|c|c|c|c|c|c|c|c|c|c|c|c|c|c|c|c|c|}
\hline & \multicolumn{17}{|c|}{4 central bits of the inputs } \\
\hline \multirow{20}{*}{$\begin{array}{l}2 \\
\text { Bit } \\
\text { s } \\
\text { Of } \\
\text { th } \\
\text { e } \\
\text { en } \\
\text { ds }\end{array}$} & & 0 & 0 & 0 & 0 & 0 & 0 & 0 & 0 & 1 & 1 & 1 & 1 & 1 & 1 & 1 & 1 \\
\hline & & 0 & 0 & 0 & 0 & 1 & 1 & 1 & 1 & 0 & 0 & 0 & 0 & 1 & 1 & 1 & 1 \\
\hline & & 0 & 0 & 1 & 1 & 0 & 0 & 1 & 1 & 0 & 0 & 1 & 1 & 0 & 0 & 1 & 1 \\
\hline & & 0 & 1 & 0 & 1 & 0 & 1 & 0 & 1 & 0 & 1 & 0 & 1 & 0 & 1 & 0 & 1 \\
\hline & & 0 & 1 & 0 & 0 & 0 & 1 & 1 & 0 & 1 & 0 & 0 & 1 & 1 & 0 & 1 & 1 \\
\hline & 0 & 0 & 1 & 1 & 0 & 1 & 0 & 0 & 1 & 0 & 1 & 0 & 1 & 1 & 0 & 1 & 0 \\
\hline & 0 & 1 & 0 & 0 & 0 & 1 & 1 & 1 & 1 & 0 & 0 & 1 & 1 & 0 & 0 & 1 & 0 \\
\hline & & 0 & 0 & 0 & 1 & 1 & 0 & 1 & 0 & 0 & 1 & 1 & 1 & 1 & 0 & 0 & 1 \\
\hline & & 1 & 1 & 0 & 1 & 0 & 0 & 1 & 0 & 0 & 0 & 1 & 1 & 0 & 1 & 1 & 0 \\
\hline & 0 & 1 & 0 & 0 & 1 & 1 & 1 & 1 & 0 & 1 & 0 & 1 & 1 & 0 & 0 & 0 & 1 \\
\hline & 1 & 1 & 1 & 1 & 0 & 0 & 1 & 0 & 0 & 0 & 0 & 1 & 0 & 1 & 0 & 0 & 1 \\
\hline & & 0 & 1 & 0 & 0 & 0 & 1 & 1 & 1 & 1 & 0 & 1 & 0 & 1 & 1 & 0 & 0 \\
\hline & & 0 & 0 & 0 & 1 & 1 & 1 & 0 & 1 & 1 & 1 & 1 & 0 & 0 & 0 & 0 & 1 \\
\hline & 1 & 1 & 0 & 0 & 0 & 1 & 1 & 1 & 0 & 1 & 0 & 1 & 1 & 1 & 0 & 0 & 1 \\
\hline & 0 & 0 & 1 & 0 & 1 & 0 & 0 & 1 & 0 & 1 & 0 & 0 & 0 & 1 & 1 & 0 & 1 \\
\hline & & 0 & 0 & 1 & 1 & 0 & 1 & 1 & 0 & 1 & 1 & 0 & 1 & 0 & 1 & 0 & 0 \\
\hline & & 1 & 1 & 1 & 0 & 0 & 1 & 0 & 1 & 0 & 1 & 0 & 1 & 1 & 0 & 0 & 0 \\
\hline & 1 & 0 & 0 & 1 & 1 & 0 & 1 & 0 & 1 & 1 & 1 & 0 & 0 & 1 & 1 & 1 & 0 \\
\hline & 1 & 1 & 0 & 0 & 1 & 0 & 1 & 1 & 0 & 1 & 1 & 0 & 0 & 0 & 0 & 0 & 1 \\
\hline & & 1 & 0 & 0 & 1 & 1 & 0 & 0 & 1 & 0 & 1 & 0 & 1 & 0 & 0 & 1 & 1 \\
\hline
\end{tabular}

Substitution is applied through eight " 8 " boxes substitutions used to reduce the size of blocks of 48 bits to 32 bits. Here's an example of an S-Box (S5). The output of 4 bits is obtained from the input of 6 bits. These 6 bits are divided in two parts: two bits at the ends and the four remaining bits (in the center). The two bits indicate the line and the central bits give the corresponding column. For example, with an input "011011" is divided into "0 11011 " Which gives for the line «01» and column «1101». The output of the table is "1001".

Figure 2. Une s-box de substitution.

We apply, in parallel, 8 boxes (fixed function) of 6 bits to 4 bits. This reduces the internal state of $8 \times 6=$ 48 bits to 8 bits $\times 4=32$ bits.

\section{3) Permutations}

Once the substitution is done a change in position of the result according to a permutation function causes a disorder of the "32" bit by the following table:

\begin{tabular}{|l|l|l|l|l|l|l|l|}
\hline 16 & 7 & 20 & 21 & 29 & 12 & 28 & 17 \\
\hline 1 & 15 & 23 & 26 & 5 & 18 & 31 & 10 \\
\hline 2 & 8 & 24 & 14 & 32 & 27 & 3 & 9 \\
\hline 19 & 13 & 30 & 6 & 22 & 11 & 4 & 25 \\
\hline
\end{tabular}

Figure 3. Permutation Table

\section{- Logic sum using XOR}

The obtained blocks composed by 32 bits are summed using the XOR logic functions with $G_{0}$ to give $D_{1}$ and the same think with the $D_{0}$ block that outputs $G_{1}$.

- Initial inverse transposition 
The initial proposed algorithm at the end of the sixteen iterations, two blocks $G_{16}$ and $D_{16}$ are "glued" to reform a single block of 64 bits then undergoes initial inverse transposition. Our algorithm offers 64 continuous iterations, which generates at the end of the round two blocks $G_{160}$ and $D_{160}$. The final text will be from gluing the two blocks. In the initial proposed algorithm and at the end of the sixteen iterations, two blocks $G_{16}$ and $D_{16}$ are "glued" to reform a single block of 64 bits then undergoes initial inverse transposition. Our algorithm offers 64 continuous iterations, which generates at the end of the round two blocks $G_{160}$ and $D_{160}$. The final text will be from joining the two blocks.

\section{- Algorithm of key generating}

The key $K$ of length 64 bits contains 8 bits for controls to ensure the integrity of information. So far its useful part is 56 bits. The key $K$ provides 160 partial key $K_{1}, \ldots ., K_{160} 48$ bits each one to be used in each of the 160 rounds that replace the 16 rounds used in the old algorithm. At first the key parity are eliminated to obtain key of 56 bits. This block then undergoes a permutation is divided into two 2 blocks of 28 bits. These two blocks are rotated to the left, that is to say that the bits in the second position take the first position, and those in the third position take the second, and so on. The blocks are grouped to a block of 56 bits that passes through a permutation providing a block of 48 bits representing the key $k_{i}$. These iterations provide the 160 keys used in the proposed algorithm.

\section{IMPLEMENTATION OF AN INTERFACE FOR MULTI- ENCRYPTION FILE}

The application of these two encryption algorithms associated together to encrypt alternatively a plain text file increases security and minimizes the possibility of deciphering the secret text. The plaintext is partitioned into subsets of words. The collected subsets are swapped in the same text with a simple offset and for each one of the subsets one between the proposed techniques is applied. The choice of the technique is based on a key is composed of two elements $K=\left\{k_{1}, k_{2}\right\} / k_{1} \in[1: 9]$ and $k_{2} \in[1: 9]$. Each element of the key indicates the number of periods with which one of the two algorithms is applied. The following figure and equations illustrate the operation of partitioning the plaintext and the implementation of encryption algorithms. In the case where $k_{1}=1$ et $k_{2}=1$ the sub-sets of the plain text are alternatively encrypted with the same period by the algorithms noted $\mathrm{AL}_{1}$ and $\mathrm{AL}_{2}$. Considering $\mathrm{M}$ the file text, $\mathrm{M}_{\mathrm{c}}$ the encrypted text, and $M_{i}$ the sub-sets, the following equation resumes the encryption step:

$$
\begin{aligned}
& M_{c}=\left\{A L_{1}\left(M_{1}\right), ., A L_{1}\left(M_{2 t}\right), A L_{2}\left(M_{2 t+1}\right)\right\} \\
& \text { such as } j=2 t+1
\end{aligned}
$$

The following figures give an overview of polyencrypting a plain text with an example of an original file and the result of its encryption.

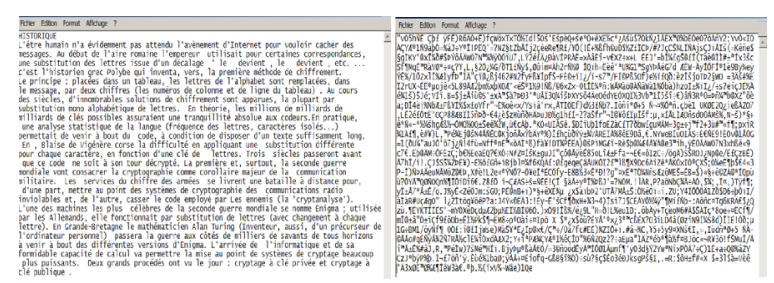

Figure 4. Plain text and its poly-encryption by LSCE and DESM with $\mathrm{k} 1=2$ et $\mathrm{k} 2=3$

\section{CONCLUSION}

In this paper, a new approach for text and text file poly-encryption is presented. This approach is composed by two techniques, the first is based on stream cipher symmetric encryption methods and the second uses a block cipher encryption structure. These two techniques are complex, the first named LSCE based on random key logic encryption independent of the text and the second named DESM inspired from the DES algorithm with a modification allowing it to be more complex and secure. These two techniques are combined to encrypt alternately a text partitioned into a set of words. Each set is encrypted by one of the two algorithms with a key that supervises the redundancy of these sets encryption. The resulting text is polyencrypted by two different techniques which make the possibility of deciphering it more difficult.

\section{REFERENCES}

[1] Schlesinger, R., "A Cryptography Course for NonMathematicians", 1st annual conference on information security curriculum development, Kennesaw, Georgia, October 08-08,2004.

[2] Maurer, .U, "The Role of cryptography in Database Security", proceeding of the 2004ACM SIGMOD international conference on Management of data (SIGMOD’04), PP5-10, ISBN: 1-58113-859-8, Paris, France June 13-18-2004.

[3] Anan, .T, Kuraki. K, Takahashi. J, "Paper Encryption Technology”, Fujitsu Sci, Tech j, Vol 46, No.1, pp.87-94, Yan. 2010

[4] Y. Mao G. Chen, S. Lian, "A Novel Fast Image Encryption Scheme Based On 3D Chaotic Baker Maps", International Journal of Bifurcation and Chaos, Vol. 14, No. 10 (2004) 3613-3624. http://dx.doi.org/10.1142/S021812740401151X

[5] Ali Bani Younes, and Jantan, .A, "Image Encryption Using Block-Based Transformation Algorithm", IAENG International Journal of Computer Science, 35:1, IJCS_35_1_03.

[6] Xu, .X Dexter, S. and Eskicioglu, A. M., "A hybrid scheme for encryption and watermarking", IEEE Journal on Selected Areas in Communications 18 (2000) 850-. 860. 13. http://dx.doi.org/10.1109/49.848239

\section{AUTHOR}

Hassene seddik is with the ENSIT school of Tunisia, Unité de recherche CEREP, hassene.seddik@esstt.rnu.tn. He is born in 15 October 1970 in Tunisia, he has abtained the electromechanical engineer degree in 1995 and followed by the master degree in "signal processing: speaker recognition" and the thesis degree in image processing "watermarking using non conventional transformations". He has over 
14 international journals papers and 65 conference papers. His domain of interest is: Audio-image and video processing applied in filtering, encryption and watermarking. He belongs to the CEREP research unit and supervises actually five thesis and 08 masters in the field.

Submitted 10 June 2013. Published as re-submitted by the author 23 July 2013 